\title{
Enhancing anti-tumor immunity through local gene delivery to lymph nodes
}

\author{
Neil M Dold*, Christopher M Jewell \\ From 30th Annual Meeting and Associated Programs of the Society for Immunotherapy of Cancer (SITC 2015) \\ National Harbor, MD, USA. 4-8 November 2015
}

\begin{abstract}
Introduction
Biodegradable polymer carriers offer attractive features for therapeutic cancer vaccines including delivery of multiple vaccine components, efficient internalization, and sustained release of adjuvants and tumor-associated antigens (TAAs). We previously demonstrated that local delivery of depots containing nucleic acid-based tollliked receptor agonists (TLRas) to lymph nodes (LNs) potently enhances antigen-specific $\mathrm{T}$ cell immunity. Building on this work, we hypothesized that local LN delivery of microparticles loaded with TAA-encoding plasmid DNA (pDNA) and TLRas might drive strong local expression and presentation of antigen by LN-resident antigen presenting cells. These effects could help drive more potent and effective CD8 $+\mathrm{T}$ cell functions that slow or stop tumor growth.
\end{abstract}

\section{Methods}

Plasmids were designed and synthesized to encode model reporter genes or a conserved $\mathrm{T}$ cell peptide epitope, TRP2 (SVYDFFVWL) for human and murine melanoma. Screening studies with primary dendritic cells utilized poly(lactic-co-glycolide) microparticles synthesized by water-oil-water emulsion, stabilized with polyvinyl alcohol. The inner aqueous phase was loaded with a TLR9 agonist (CpG oligodeoxynucleotide) and pDNA. Size and loading were characterized by laser diffraction and UV-vis spectroscopy. Murine splenocytes expressing CD11c were harvested, isolated, and treated overnight in triplicate with empty particles or particles encapsulating CpG only, pDNA only, or both CpG+pDNA. Cells were analyzed by flow cytometry using markers for viability (DAPI-) and dendritic cell activation (CD40+, CD80+, CD86+).

\footnotetext{
Fischell Department of Bioengineering, University of Maryland - College Park,
} College Park, MD, USA

\section{Results}

The pDNA vector for the TRP2 epitope was designed to express degradable peptide spacers (AAY) flanking repeating units of the antigenic epitope. This vector was synthesized with and without a sequence encoding ubiquitin to provide efficient proteasomal peptide processing and antigen expression. Particles exhibited mean diameters $2.5-4.4 \mu \mathrm{m}$ depending on cargo and synthesis energy. Cargo loading was measured for $\mathrm{CpG}$ particles at $3.8 \mu \mathrm{g} \mathrm{CpG} / \mathrm{mg}$ particles and for pDNA particles at $11.1 \mu \mathrm{g} \mathrm{pDNA} / \mathrm{mg}$ particles. During ex vivo screening, particles containing cargo increased CD40, CD80, and CD86 expression compared to empty particles and did not impact cell viability compared to controls treated with soluble TLRas.

\section{Discussion}

Initial studies demonstrate microparticles loaded with TLRa and pDNA effectively stimulate primary immune cells, supporting a platform for loading and driving local levels in primary cells and secondary lymph organs using a mouse melanoma model and will correlate local TAA expression with antigen-specific $T$ cell expansion and efficacy during tumor challenge.

\section{Acknowledgements}

\#15051543.

Published: 4 November 2015

doi:10.1186/2051-1426-3-S2-P431

Cite this article as: Dold and Jewell: Enhancing anti-tumor immunity through local gene delivery to lymph nodes. Journal for Immunotherapy of Cancer 2015 3(Suppl 2):P431. 